\title{
Constitutive androstane receptor activation promotes bilirubin clearance in a murine model of alcoholic liver disease
}

\author{
XIUYAN WANG ${ }^{1,2}$, LIYU ZHENG $^{2}$, JINMING WU $^{2}$, BINBIN TANG $^{2}$, \\ MENGQIN ZHANG ${ }^{2}$, DEBIN ZHU ${ }^{2}$ and XIANFAN LIN ${ }^{2}$ \\ ${ }^{1}$ Department of Gastroenterology, Wenzhou People's Hospital; ${ }^{2}$ Department of Gastroenterology, \\ The First Affiliated Hospital of Wenzhou Medical University, Wenzhou, Zhejiang 325000, P.R. China
}

Received December 2, 2015; Accepted November 29, 2016

DOI: $10.3892 / \mathrm{mmr} .2017 .6435$

\begin{abstract}
Increased plasma levels of bilirubin have been reported in rat models and patients with alcoholic liver disease (ALD). The constitutive androstane receptor (CAR) is a known xenobiotic receptor, which induces the detoxification and transport of bilirubin. In the present study, the bilirubin transport regulatory mechanisms, and the role of CAR activation in hepatic and extrahepatic bilirubin clearance were investigated in a murine model of ALD. The mice were fed a Lieber-DeCarli ethanol diet or an isocaloric control diet for 4 weeks, followed by the administration of CAR agonists, 1,4-bis-[2-(3,5-dichlorpyridyloxy)]benzene (ТCРОВOP) and phenobarbital (PB), and their vehicles to examine the effect of the pharmacological activation of CAR on serum levels of bilirubin and on the bilirubin clearance pathway in ALD by serological survey, western blotting and reverse transcription-quantitative polymerase chain reaction. The results showed that chronic ethanol ingestion impaired the nuclear translocation of CAR, which was accompanied by elevated serum levels of bilirubin, suppression of the expression of hepatic and renal organic anion transporting polypeptide (OATP) 1A1 and hepatic multidrug resistance-associated protein 2 (MRP2), and induction of the expression of UDP-glucuronosyltransferase (UGT) 1A1. The activation of CAR by TCPOBOP and PB resulted in downregulation of the serum levels of bilirubin followed by selective upregulation of the expression levels of OATP1A1, OATP1A4, UGT1A1 and MRP2 in ALD. These results revealed the bilirubin transport regulatory mechanisms and highlighted the importance of CAR in modulating the bilirubin clearance pathway in the ALD mouse model.
\end{abstract}

Correspondence to: Professor Jinming Wu, Department of Gastroenterology, The First Affiliated Hospital of Wenzhou Medical University, No. 2 Fu Xue Road, Wenzhou, Zhejiang 325000, P.R. China

E-mail: wzfywjm@163.com

Key words: alcoholic liver disease, bilirubin, constitutive androstane receptor, Lieber-DeCarli ethanol diet

\section{Introduction}

Alcoholism is a global social and medical problem, and the accompanying alcoholic liver disease (ALD) is one of the most common causes of chronic liver disease, and contributor to morbidity and mortality rates worldwide. ALD encompasses a series of conditions ranging from simple steatosis through to steatohepatitis and fibrosis, cirrhosis and hepatocellular carcinoma (1).

Bilirubin, a major end-product of the breakdown of heme, is widely used for the evaluation of ALD severity (2). Increased levels of bilirubin in the plasma have been reported in ALD rats and in patients with different stages of ALD without bile duct obstruction (3-5). Accumulated bilirubin further aggravates liver injury via the inhibition of various enzyme systems, RNA synthesis and protein synthesis, and via the uncoupling of oxidative phosphorylation in liver mitochondria (6). The dysfunction of bilirubin transportation contributes to the development of hyperbilirubinemia or cholestatic liver diseases (7-9). Under normal conditions, unconjugated bilirubin enters hepatocytes via facilitated diffusion, which is mediated by the organic anion transporting polypeptide (OATP) family of proteins expressed on the basolateral membrane, and is extensively glucuronidated by UDP-glucuronosyltransferase (UGT1A1). Finally, the glucuronide metabolite is excreted into the bile via multidrug resistance-associated protein 2 (MRP2) localized on the canalicular plasma membrane (7-9). OATP1B2, OATP1A1 and OATP1A4 in the mouse liver mediate uptake (10). Mutations of these transporter genes cause hereditary hyperbilirubinemia in humans, further suggesting that altered transporter expression or function is pivotal in the pathogenesis of cholestasis, including Rotor syndrome and Dubin-Johnson syndrome $(8,9)$. Transport regulatory mechanisms have been investigated in bile duct ligation (11), lipopolysaccharide-induced cholestasis and in a rhesus-rotavirus-induced biliary atresia mouse model (12). However, few studies have systematically investigated these mechanisms in a murine model of ALD.

The constitutive androstane receptor (CAR; NR1I3), known as a xenobiotic receptor, has been implicated in the detoxification and transportation of bilirubin or bile acid, the regulation of drug metabolism, lipid metabolism, cholesterol biosynthesis, cytokine and insulin signaling, the modulation of 
apoptosis, and tumor development via the transcriptional regulation of downstream target genes $(11,13-15)$. CAR target genes comprise those encoding phase II enzymes, including UGT isoforms (UGT1A1, UGT1A6 and UGT1A9), uptake/efflux transporters, including OATPs (OATP1, OATP2 and OATP4) and MRPs (MRP2, MRP3 multidrug resistance protein 1 (MDR1), cytochrome P450 (CYP) 2B6, CYP2C9, CYP3A4, glutathione S-transferase (GST) A1 and GSTA2 (14,16-18). Among these target genes, UGT1A1, OATPs and MRPs are key components of the bilirubin clearance pathway, thus CAR has a regulatory role in bilirubin clearance.

The elevated bilirubin levels in ALD and the role of CAR in bilirubin clearance raise the question of whether ethanol alters the expression of components of the bilirubin transport systems or detoxifying enzymes via CAR. The present study investigated the expression of different components of bilirubin clearance and of the xenobiotic receptor, CAR. In addition, the role of CAR activation in the hepatic and renal bilirubin clearance pathway of a murine model of ALD was investigated using the direct ligand, 1,4-bis-[2-(3,5-dichlorpyridyloxy)] benzene (TCPOBOP), and the indirect ligand, phenobarbital (PB), which does not bind to the receptor but translocates CAR to the nucleus $(18,19)$.

\section{Materials and methods}

Chemicals. TCPOBOP was purchased from Sigma-Aldrich; Merck Millipore (Darmstadt, Germany), PB was purchased from Spectrum (Scottsdale, NJ, USA) and dimethyl sulfoxide (DMSO) was purchased from Sigma-Aldrich; Merck Millipore.

Animals and treatment. A total of 48 male mice (8-10 weeks old; 20-25 g) on a C57BL/6 J background were purchased from Shanghai Laboratory Animal Center, Chinese Academy of Sciences (Shanghai, China) and housed in a specific pathogen-free animal facility under constant temperature $\left(20-22^{\circ} \mathrm{C}\right)$, controlled humidity $(45-55 \%)$ and a standard $12-\mathrm{h}$ light/dark cycle. All experimental procedures were approved by the Institutional Animal Committee of Wenzhou Medical University (Wenzhou, China) and performed according to the Guide for the Care and Use of Laboratory Animals (Wenzhou Medical University, Wenzhou, China).

The regular Lieber-DeCarli ethanol liquid diet (ETOH group) or an isocaloric control diet (pair-fed group) were purchased from Trophic Animal Feed High-tech Co., Ltd. (Nantong, China), and were fed to the male mice ad libitum, as previously described (20). The percentages of caloric intake from ethanol (maltose dextrin), proteins, carbohydrates and fats were $35.5,18,11.5$ and $35 \%$, respectively. In the first week, mice were gradually introduced to the ethanol liquid diet containing $0.75 \%$ ethanol (w/v) for 2 days, $1.50 \%$ ethanol (w/v) for 2 days and $3.75 \%$ ethanol (w/v) for 3 days to acclimatize the mice to the liquid diet, and were then introduced to a $5 \%$ ethanol (w/v) diet for 4 weeks. No differences in body weight or the quantity of food consumed were found among the treated groups. In the following 3 days, the clinically used CAR ligand, PB (100 mg/kg/d; dissolved in DMSO) and the specific rodent CAR ligand, TCPOBOP $(3 \mathrm{mg} / \mathrm{kg} / \mathrm{d}$; dissolved in corn oil), which has a higher potency, were administered intraperitoneally (i.p) to the mice in the ETOH group (11). As a vehicle, mice were injected i.p with $100 \mu \mathrm{l}$ of DMSO (ETOH/DS group) or corn oil (ETOH/CO group) for comparison with the ETOH/PB and ETOH/TCP groups, respectively. A total of four animals were investigated in each treatment group. A total of 3 days following injection, the animals were anesthetized with $5 \%$ chloral hydrate $(40 \mathrm{mg} / \mathrm{kg}$, i.p) and sacrificed using the cervical dislocation method following blood collection. Blood was collected from the orbital sinus of the animals by traumatic avulsion of the globe from the orbit using a pair of tissue forceps. Sections of liver tissue were fixed in $4 \%$ paraformaldehyde solution and paraffin-embedded for light microscopy. In addition, liver tissues were cut into sections measuring $<5 \mathrm{~mm}$ in thickness, embedded in OCT compound and frozen in liquid nitrogen. The remaining liver samples and the serum samples were stored at $-80^{\circ} \mathrm{C}$ for further analysis.

Liver sectioning and histological staining. The paraformaldehyde-fixed, paraffin-embedded liver samples were cut into $5-\mu$ m-thick sections and the OCT compound-embedded liver samples were cut into $8-\mu \mathrm{m}$-thick frozen sections, respectively. A light microscope was used to visualize hematoxylin and eosin staining, as well as Oil Red O (Sigma-Aldrich; Merck Millipore) staining to quantify steatosis and inflammation in each group.

Serum biochemistry. Serum was prepared from the blood by centrifugation at $1,200 \mathrm{~g}$ at $4^{\circ} \mathrm{C}$ for $10 \mathrm{~min}$. The levels of total serum bilirubin (TB), conjugated bilirubin (CB), alanine aminotransferase (ALT), aspartate aminotransferase (AST) and alkaline phosphatase (ALP) were determined in the pair-fed, ETOH, ETOH/PB, ETOH/TCP, $\mathrm{ETOH} / \mathrm{CO}$ and ETOH/DS mice using a total/direct bilirubin kit (Sigma-Aldrich; Merck Millipore) and an ALT/AST assay kit (Jiancheng Bioengineering Institute, Nanjing, China) according to the respective manufacturers' protocols.

Western blot analysis. For isolation of total liver and kidney membranes, tissue was homogenized in $1 \mathrm{mM} \mathrm{NaHCO}$ ( $\mathrm{pH}$ 7.4), containing complete protease inhibitor (Beyotime Institute of Biotechnology, Haimen, China). Homogenates were gauze filtered, and total membranes were isolated by centrifugation at $100,000 \times \mathrm{g}$ for $1 \mathrm{~h}$ at $4^{\circ} \mathrm{C}$. as previously described $(12,21)$. Nuclear protein was extracted using the CelLytic $^{\mathrm{TM}}$ NuCLEAR ${ }^{\mathrm{TM}}$ Extraction kit (Sigma-Aldrich; Merck Millipore). In addition, protein concentrations were determined using a BCA protein Assay kit (Beyotime Institute of Biotechnology). A total of $20 \mu \mathrm{g}$ protein was loaded to each well of $10 \%$ polyacrylamide gels for electrophoresis. Proteins were blotted onto a polyvinylidene fluoride (PVDF) membrane (Merck Millipore). PVDF membranes were blocked using TBS containing $0.1 \%$ Tween 20 and $4 \%$ skim milk powder. Blots were incubated with polyclonal rabbit anti-mouse lamin-B (dilution, 1:5,000; cat. no. ab-65986; Abcam, Cambridge, UK), OATP1A1 (dilution, 1:1,000; cat. no. sc-47265; Santa Cruz Biotechnology, Inc., Dallas, TX, USA), OATP1A4 (dilution, 1:1,000; cat. no. sc-33610; Santa Cruz Biotechnology, Inc.), OATP1B2 (dilution, 1:1,000; cat. no. sc-134460, Santa Cruz Biotechnology. Inc.), MRP2 (dilution, 1:2,000; cat. no. sc-20766, Santa Cruz Biotechnology, Inc.), CAR (dilution, 1:1,000; cat. no. ab-186869; Abcam), 
GAPDH (dilution, 1:1,000; cat. no. AG019; Beyotime Institute of Biotechnology) primary antibodies or monoclonal goat anti-mouse UGT1A1 primary antibody (dilution, 1:1,000; cat. no. sc-27419; Santa Cruz Biotechnology, Inc.) at $4^{\circ} \mathrm{C}$ overnight, respectively. Following washing with $1 \mathrm{X}$ TBS containing $0.1 \%$ Tween 20 for 3 times, there was incubation conducted using horseradish peroxidase-conjugated goat anti-rabbit secondary antibody (dilution, 1:10,000; cat. no. A0208; Beyotime Institute of Biotechnology) and enhanced chemiluminescence substrate reagent (Thermo Fisher Scientific, Inc., Waltham, MA, USA). The time for secondary antibody incubation was $<1 \mathrm{~h}$ at $37^{\circ} \mathrm{C}$. The quantification of protein expression was determined via image processing and analysis using Image Lab 3.0 software (Bio-Rad Laboratories, Inc., Hercules, CA, USA).

Total RNA isolation and reverse transcription-quantitative polymerase chain reaction (RT-qPCR) analysis. RNA was extracted from the frozen liver and kidney samples using TRIzol reagent (Invitrogen; Thermo Fisher Scientific, Inc.) and complementary DNA (cDNA) synthesis for qPCR analysis was performed using a commercial kit (Toyobo Co., Ltd., Osaka, Japan) with subsequent melting curve analysis, according to the manufacturer's protocol. The PCR reaction mixture (forward primer, $0.4 \mu \mathrm{l}$; reverse primer, $0.4 \mu \mathrm{l}$; cDNA, $1 \mu \mathrm{l}$; diethylpyrocarbonate, $3.2 \mu \mathrm{l}$ ) was prepared using SYBR Green Real-time PCR Master Mix-Plus (Toyobo Co., Ltd.). The thermocycling parameters were as follows: pre-denaturation at $94^{\circ} \mathrm{C}$ for $1 \mathrm{~min}$, denaturation at $95^{\circ} \mathrm{C}$ for $15 \mathrm{sec}$, annealing at $60^{\circ} \mathrm{C}$ for $15 \mathrm{sec}$, extension at $72^{\circ} \mathrm{C}$ for $15 \mathrm{sec}$ for 40 cycles of amplification. PCR amplification was performed using specific primers (Table I). Data were analyzed using Bio-Rad CFX manager (Bio-Rad Laboratories, Inc.). The levels of gene expression were calculated as a ratio to the expression of the housekeeping gene, $\beta$-actin.

Immunofluorescence analysis. The NCTC1469 normal mouse liver cell line was purchased from Zhongqiao (Shanghai, China). The cells were maintained at $37^{\circ} \mathrm{C}$ in a humidified atmosphere of 5\% $\mathrm{CO}_{2}$ in RPMI 1640 supplemented with penicillin/streptomycin (100 IU/ml and $100 \mu \mathrm{g} / \mathrm{ml}$, respectively) and $10 \%$ fetal bovine serum (Gibco; Thermo Fisher Scientific, Inc.). Prior to the experiment, cell viability was measured via Trypan blue exclusion. The hepatocytes were plated on glass coverslips at a density of $2.0 \times 10^{5}$ cells $/ \mathrm{ml}$ in 6 wells and incubated in a $\mathrm{CO}_{2}$ incubator for $3 \mathrm{~h}$ at $37^{\circ} \mathrm{C}$. The cells were pretreated with or without $2 \mathrm{mM}$ phenobarbital (PB) for $6 \mathrm{~h}$ followed by treatment with or without $100 \mu \mathrm{M}$ alcohol for $12 \mathrm{~h}$ at $37^{\circ} \mathrm{C}$. The cells were fixed with $4 \%$ formaldehyde/PBS for 15 min. 10\% Goat serum (Gibco; Thermo Fisher Scientific, Inc.) in TBS containing $0.1 \%$ Triton $\mathrm{X}-100$ was used to block nonspecific binding, as previously described (22). Membranes were incubated with CAR primary antibody at a dilution of 1:100 overnight and with corresponding secondary antibodies conjugated to Delight 594 (Bioworld Technology, Inc., Minneapolis, MN, USA) at a dilution of 1:200 for another $2 \mathrm{~h}$ at room temperature. The coverslips were sealed with antifade mounting medium (Beyotime Institute of Biotechnology) following staining with DAPI (Beyotime, Haimen; China) for $10 \mathrm{~min}$, and were visualized using a fluorescence microscope (Olympus, Tokyo, Japan).
Statistical analysis. Data are shown as the mean \pm standard deviation. Statistical analysis was performed using two-tailed Student's $t$-test or one-way analysis of variance followed by post hoc tests (LSD-t or Dunnett's T3) for multigroup comparisons. SPSS 12.0 program (SPSS, Inc., Chicago, IL, USA) was used for statistical analysis. $\mathrm{P}<0.05$ was considered to indicate a statistically significant difference.

\section{Results}

Biochemical and histopathological changes in the experimental ALD mouse model. To investigate the role of CAR in bilirubin clearance in ALD, wild-type C57BL6 mice were fed with a Lieber-DeCarli liquid diet or a pair-fed control diet for 4 weeks, followed by i.p. injections with different CAR ligands for 3 days. The levels of ALT, AST and ALP in the EtOH group were higher, compared with those in the pair-fed controls $(105 \pm 30.66$, vs. $26.75 \pm 2.99 \mathrm{U} / 1 ; 187.8 \pm 55.34$, vs. $111.3 \pm 19.52 \mathrm{U} / 1$ and $155 \pm 12.83$, vs. $65.25 \pm 37.35 \mathrm{U} / 1$, respectively; $\mathrm{P}<0.05$ ) and were also elevated in the EtOH/TCP group, compared with those in its vehicle $(\mathrm{EtOH} / \mathrm{CO})$ group $(510.3 \pm 151.2$, vs. $103.8 \pm 25.63 \mathrm{U} / 1 ; 287.8 \pm 91.09$, vs. $174.5 \pm 42.15 \mathrm{U} / 1$ and $284.5 \pm 46.65$, vs. $148.8 \pm 13.74 \mathrm{U} / 1$, respectively; $\mathrm{P}<0.05$ ), as shown in Table II. The levels of AST and ALP in the EtOH/DS group (EtOH/PB vehicle) were lower, compared with those in the EtOH group $(115 \pm 25.59$, vs. $187.8 \pm 55.34 \mathrm{U} / 1$ and 106 \pm 18.94 , vs. $155 \pm 12.83 \mathrm{U} / 1$, respectively; $\mathrm{P}<0.05)$. Compared with the pair-fed controls, the levels of hepatic total bilirubin and direct bilirubin were significantly elevated following alcohol ingestion $(0.33 \pm 0.10$, vs. $0.75 \pm 0.17 \mu \mathrm{mol} / 1$ and $0.25 \pm 0.10$, vs. $0.45 \pm 0.13 \mu \mathrm{mol} / 1$, respectively; $\mathrm{P}<0.05)$, and were markedly decreased following administration of the high affinity CAR agonist, TCPOBOP $(0.55 \pm 0.13$, vs. $0.15 \pm 0.06 \mu \mathrm{mol} / 1$ and $0.43 \pm 0.10$, vs. $0.13 \pm 0.05 \mu \mathrm{mol} / 1$, respectively; $\mathrm{P}<0.05)$. The level of total bilirubin was reduced following indirect ligand PB injection whereas only a marginal alteration was observed in the level of direct bilirubin (Table II). Furthermore, the levels of total and direct bilirubin in the EtOH/DS group (EtOH/PB vehicle) were downregulated, compared with those in the EtOH group $(0.45 \pm 0.06$, vs. $0.75 \pm 0.17 \mu \mathrm{mol} / 1$ and $0.2 \pm 0.08$, vs. $0.45 \pm 0.13 \mu \mathrm{mol} / 1$, respectively; $\mathrm{P}<0.05)$. Taken together, chronic alcohol consumption led to ALD, and increased the levels of total and direct bilirubin. In addition, the CAR agonists, TCPOBOP and PB, decreased total and direct bilirubin levels.

$\mathrm{H} \& \mathrm{E}$ staining and oil red $\mathrm{O}$ staining revealed that chronic alcohol ingestion caused liver macrovesicular and microvesicular steatosis, cellular edema and ballooning degeneration, and nuclear pycnosis. These liver injuries were ameliorated by the CAR ligands, TCPOBOP and PB (Fig. 1A and B). In addition, TCPOBOP treatment resulted in hepatocyte enlargement, based on an elevated liver/body weight ratio $(\mathrm{P}<0.001$; Fig. 1C). Chronic alcohol intake also significantly dysregulated the liver/body weight ratio, as shown in Fig. $1 \mathrm{C}(\mathrm{P}<0.01)$.

Effect of chronic alcohol consumption on hepatic and extrahepatic bilirubin clearance. The mRNA and protein levels of the primary mouse hepatic bilirubin uptake transporters, OATP1A4 and OATP1B2, remained unaltered following 4 weeks of chronic alcohol consumption (Fig. 2A and C). 
Table I. Mouse primers.

\begin{tabular}{lll}
\hline Gene & \multicolumn{1}{c}{ Forward primer $\left(5^{\prime}-3^{\prime}\right)$} & \multicolumn{1}{c}{ Reverse primer (5'-3') } \\
\hline Oatp1a1 & GTCTTACGAGTGTGCTCCAGAT & GGAATACTGCCTCTGAAGTGGATT \\
Oatp1a4 & GACGGCTCAGTGTTCATTC & CTTCTAGCTGGTCCCTCTT \\
Oatp1b2 & GATCCTTCACTTACCTGTTCAA & CCTAAAAACATTCCACTTGCCATA \\
Mrp2 & GCTTCCCATGGTGATCTCTT & ATCATCGCTTCCCAGGTACT \\
Ugt1a1 & TCTGAGCCCTGCATCTATCTG & CCCCAGAGGCGTTGACATA \\
Car & AAAGCAGGGTCAGCGAGGAG & AGTCAGGGCGTGGAAATGATAGC \\
$\beta$-actin & CTGGCACCACACCTCCTACA & AGTACTTGCGCACAG GAGGA
\end{tabular}

Reverse transcription-quantitative polymerase chain reaction analysis was performed using $\mathrm{SYBR}^{\circledR}$ Green. Car, constitutive androstane receptor; Oatp, organic anion transporting polypeptide; Mrp, multidrug resistance-associated protein; Ugt, UDP-glucuronosyltransferase.

Table II. Serum biochemistry.

\begin{tabular}{lcccccc}
\hline Characteristic & Pair-fed & EtOH & EtOH/TCP & EtOH/CO & EtOH/PB & EtOH/DS \\
\hline Total bilirubin $(\mu \mathrm{mol} / \mathrm{l})$ & $0.33+0.10^{\mathrm{a}}$ & $0.75+0.17$ & $0.15+0.06^{\mathrm{b}}$ & $0.55+0.13$ & $0.23+0.05^{\mathrm{b}}$ & $0.45+0.06^{\mathrm{a}}$ \\
Direct bilirubin $(\mu \mathrm{mol} / \mathrm{l})$ & $0.25+0.10^{\mathrm{a}}$ & $0.45+0.13$ & $0.13+0.05^{\mathrm{b}}$ & $0.43+0.10$ & $0.10+0.08$ & $0.2+0.08^{\mathrm{a}}$ \\
ALT (U/l) & $26.75+2.99^{\mathrm{a}}$ & $105+30.66$ & $510.3+151.2^{\mathrm{b}}$ & $103.8+25.63$ & $51.5+12.92$ & $79.3+19.41$ \\
AST (U/l) & $111.3+19.52^{\mathrm{a}}$ & $187.8+55.34$ & $287.8+91.09^{\mathrm{b}}$ & $174.5+42.15$ & $125.3+29.3$ & $115+25.59^{\mathrm{a}}$ \\
ALP (U/l) & $65.25+37.35^{\mathrm{a}}$ & $155+12.83$ & $284.5+46.65^{\mathrm{b}}$ & $148.8+13.74$ & $86+27.06$ & $106+18.94^{\mathrm{a}}$ \\
\hline
\end{tabular}

Serum biochemistry was assessed in each group. ${ }^{a} \mathrm{P}<0.05$ between ethanol and control or vehicle. ${ }^{b} \mathrm{P}<0.05$ between treatment group and vehicle ALT, alanine aminotransferase; AST, aspartate aminotransferase; ALP, alkaline phosphatase; TCP, 1,4-bis-[2-(3,5-dichlorpyridyloxy)]benzene; $\mathrm{CO}$, corn oil; $\mathrm{PB}$, phenobarbital; DS, dimethyl sulfoxide.

However, the mRNA level of hepatic OATP1A1 in the EtOH group was markedly reduced by $\sim 60$-fold (Fig. $2 \mathrm{~A}$ ). The protein levels of OATP1A1 were decreased by $60 \%$, compared with those of the pair-fed group (Fig. 2C), in accordance with the downregulated mRNA levels (Fig. 2A). Furthermore, alcohol intake induced the mRNA and protein expression of bilirubin-glucuronidating UGT1A1 4.9- and 1.5-fold (Figs. 2B and C), respectively, as previously observed in a rat model of ALD (5). The mRNA levels of the excretion pump, MRP2, were significantly decreased 2.2-fold (Fig. 2B), whereas the protein level of MRP2 was deregulated ( $\mathrm{P}<0.05$; Fig. $2 \mathrm{C}$ ), compared with that of the pair-fed group.

OATP1A1, OATP1A4 and MRP2 also have been detected in the apical membrane of renal proximal tubular cells $(10,17,23,24)$. Under pathological conditions, including cholestasis, bilirubin glucuronides are secreted into the plasma and then eliminated by the kidney (10). Adaptive renal transporter induction has been confirmed in mouse cholestatic models of common bile duct ligation (23). To investigate the extrahepatic pathway of bilirubin clearance in the ALD mouse model, the present study examined the renal expression of these transporters. The mRNA and protein expression levels of OATP1A1 in the kidney were decreased by 25 - and 1.6-fold, respectively, compared with those in the pair-fed group (Figs. 2D and E). The expression levels of renal OATP1A4 and MRP2 remained unaffected (Figs. 2D and E). Previous reports on renal bilirubin transport in an ALD mouse model are limited.
Effect of chronic alcohol consumption on the expression of nuclear receptor CAR. The present study also examined whether the expression of CAR was affected by chronic alcohol ingestion. The alcohol-fed mice showed limited variation in mRNA and protein levels of CAR in livers of the alcohol-fed mice, whereas the protein level of CAR in nuclear extracts was negatively regulated by $60 \%$, compared with that of the pair-fed group (Fig. 2B and F), suggesting that alcohol inhibited CAR translocation to the nucleus.

Effect of the TСРОВOP and $P B$ on hepatic and extrahepatic bilirubin clearance in a murine model of $A L D$. To determine the role of the TCPOBOP and PB CAR ligands in the regulation of bilirubin clearance in ALD mice, TCPOBOP, PB and their vehicles were administered (i.p.). A marked increase was observed in the mRNA expression levels of liver Oatpla1, Oatpla4 and Ugtla1 in the EtOH/PB group, compared with that of the vehicle EtOH/DS group (2.5-, 1.3- and 4.6-fold, respectively) whereas no significant change in the levels of Oatp1b2 or Mrp2 were observed (Fig. 3A and B). An increase in the mRNA expression of Mrp2 of >2-fold was observed in the EtOH/TCP group, compared with its vehicle $\mathrm{EtOH} / \mathrm{CO}$ group, whereas the mRNA levels of Oatp1a1, Oatp1a4, Oatp1b2 and Ugt1a1 were not altered significantly (Fig. 3A and B). Accordingly, the protein levels of UGT1A1 in the liver were induced following treatment with TCPOBOP and $\mathrm{PB}$, compared with the levels in their vehicles (1.5- and 2-fold, respectively, $\mathrm{P}<0.05$; Fig. 3C). 
A
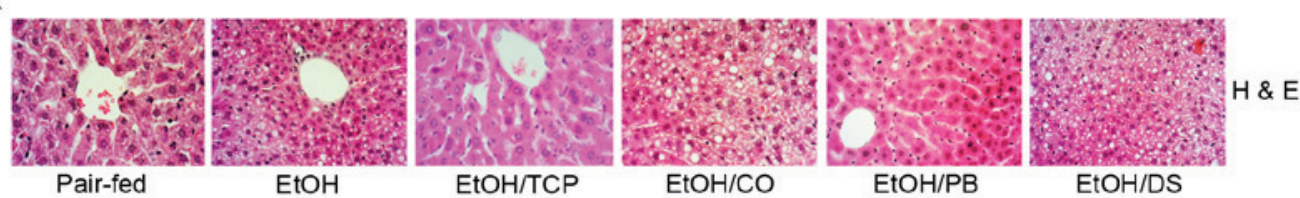

B
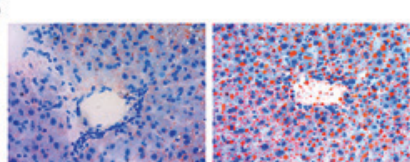

Pair-fed $\mathrm{EtOH}$

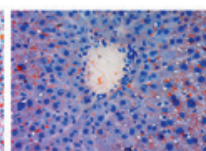

$\mathrm{EtOH} / \mathrm{TCP}$

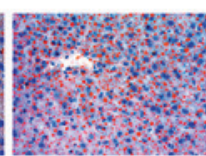

$\mathrm{EtOH} / \mathrm{CO}$

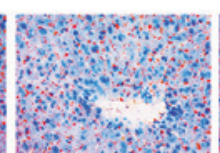

$\mathrm{EtOH} / \mathrm{PB}$
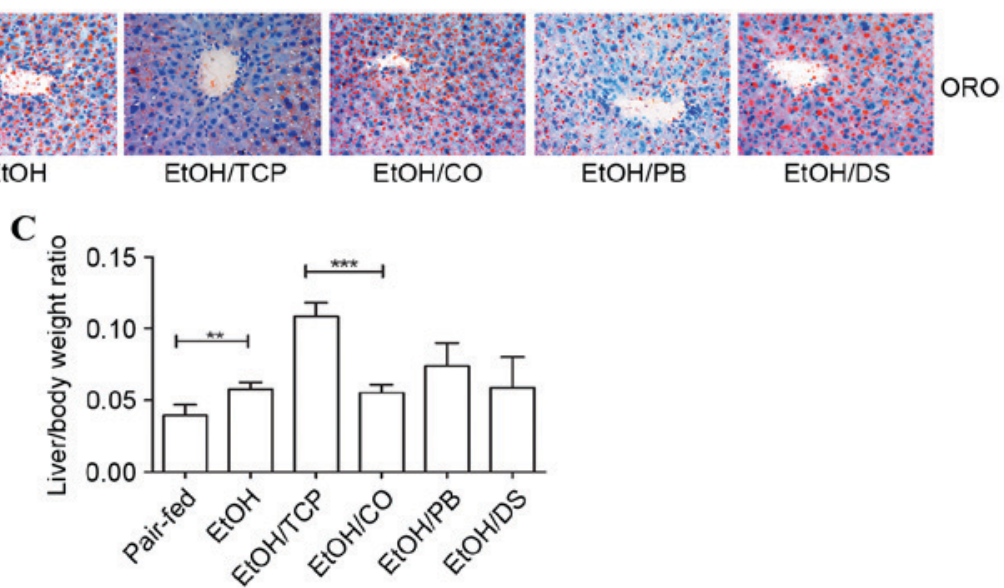

Figure 1. Effect of CAR agonists, TCP and PB, and their vehicles, corn oil and dimethyl sulfoxide, on alcohol-induced fatty liver in mice. (A) Liver sections were stained with H\&E (original magnification, x400). (B) ORO staining of the liver sections showing the number of oil drops in the sections from each group (original magnification, $\mathrm{x} 400$ ). (C) Liver/body weight ratio in each group. Scale bar $=50 \mu \mathrm{m}$. $\left(\mathrm{n}=4,{ }^{* * *} \mathrm{P}<0.01\right.$ and $\left.{ }^{* * * *} \mathrm{P}<0.001\right)$. CAR, constitutive androstane receptor; PB, pentobarbital; TCP, 1,4-bis-[2-(3,5-dichlorpyridyloxy)]benzene; CO, corn oil; DS, dimethyl sulfoxide; H\&E, hematoxylin and eosin; ORO, oil red $\mathrm{O}$.

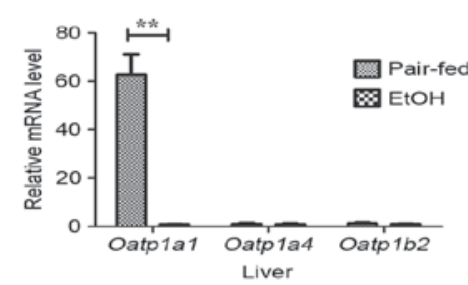

D

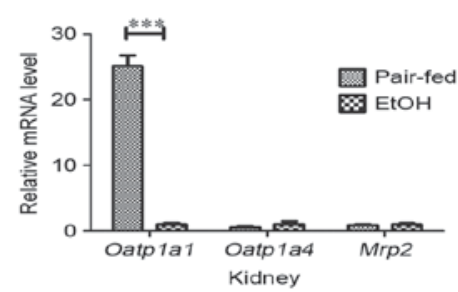

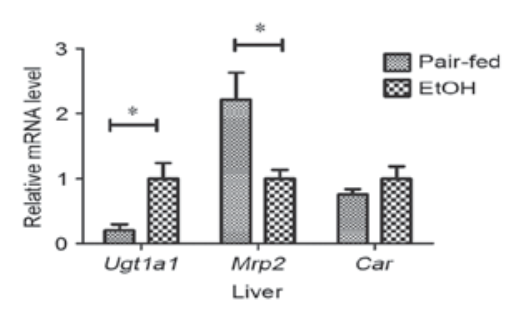

$\mathrm{E}$

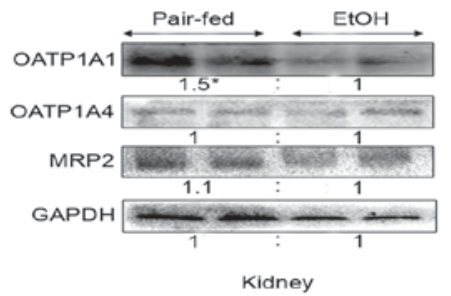

$\mathrm{C}$

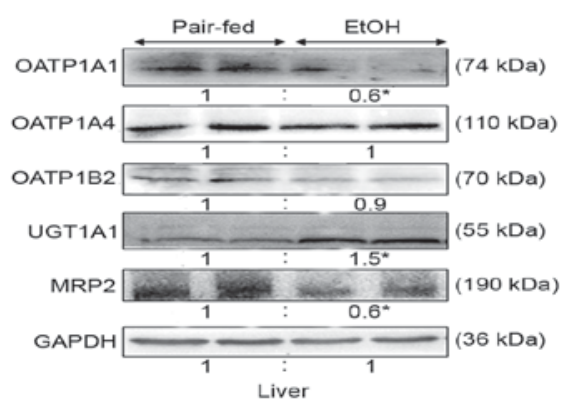

$\mathrm{F}$

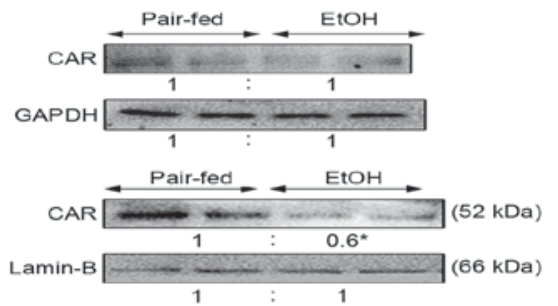

Figure 2. Effects of chronic alcohol consumption on bilirubin uptake, detoxification and excretion. (A) mRNA levels of OATP1a1, OATP1a4 and OATP1b2 uptake transporters in mouse livers were assayed following 4 weeks on an alcoholic diet. (B) Western blotting analysis of protein expression of liver OATP1a1, OATP1a4, OATP1b2, UGT1A1 and MRP2. (C) mRNA levels of liver detoxification and excretion enzymes, UGT1A1 and MRP2, in mice. (D) Nuclear extracts from liver and liver membrane were subjected to western blot analysis to determine the protein level of CAR. (E) Kidney tissue was subjected to reverse transcription-quantitative polymerase chain reaction analysis for determination of mRNA levels of OATP1a1, OATP1a4 and MRP2. (F) Western blot analysis of OATP1a1, OATP1a4 and MRP2 in the kidney ( $\mathrm{n}=4$ per group; ${ }^{*} \mathrm{P}<0.05,{ }^{* *} \mathrm{P}<0.01$ and $\left.{ }^{* * *} \mathrm{P}<0.001\right)$. CAR, constitutive androstane receptor; OATP, organic anion transporting polypeptide; UGT, UDP-glucuronosyltransferase; MRP, multidrug resistance-associated protein.

MRP2 was also increased significantly in the EtOH/TCP group (1.4-fold; $\mathrm{P}<0.05)$ and marginally induced in the EtOH/PB group (1.3-fold; $\mathrm{P}>0.05$; Fig. $3 \mathrm{C}$ ). No significant enhancement in the relative protein levels of OATP1A1, OATP1A4 or OATP1B2 were found in the EtOH/TCP or EtOH/PB groups (Fig. 3C).
To further elucidate the effect of CAR ligands on the extrahepatic bilirubin clearance pathway in ALD mice, the present study detected the renal expression of OATP1A1, OATP1A4 and MRP2 transporters. The mRNA levels of Oatpla1 and Oatpla4 remained unchanged following treatment with the 
A

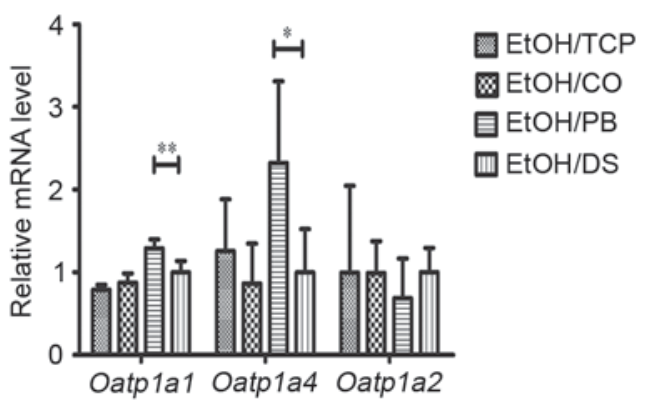

C

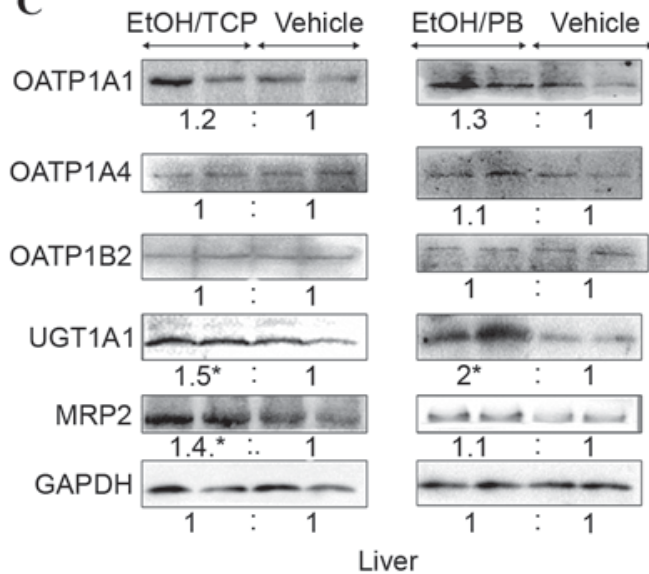

$\mathbf{E}$

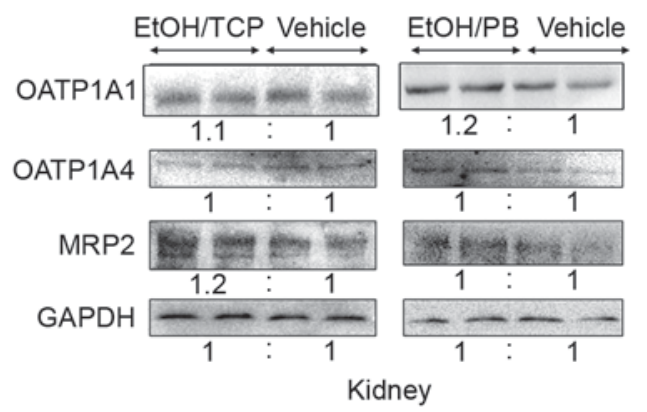

B

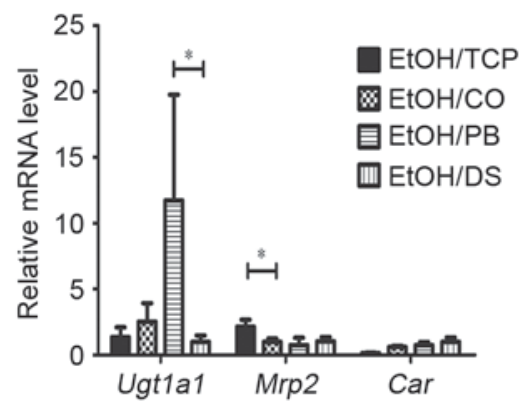

D

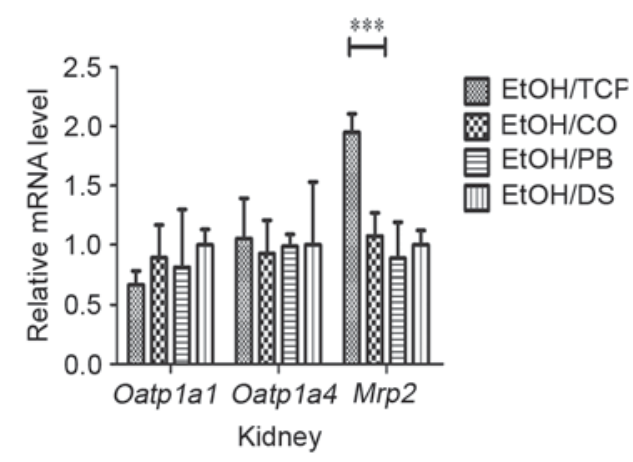

F

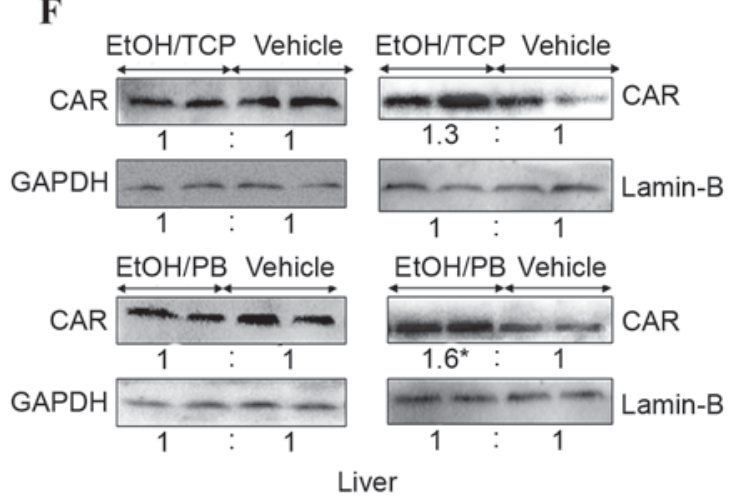

Figure 3. TCP and PB selectively increase the expression of bilirubin-detoxifying enzymes, transporters and CAR in the liver and kidney. (A) RT-qPCR analysis of total liver mRNA expression of Oatp1a1, Oatpla4 and Oatp1b2. (B) RT-qPCR analysis of total liver mRNA expression of Ugt1A1, Mrp2 and Car. (C) Western blot analysis of liver protein expression levels of OATP1A1, OATP1A4, OATP1B2, UGT1A1 and MRP2. (D) RT-qPCR analysis of renal mRNA levels of Oatp1a1, Oatpla4, Mrp2. (E) Western blot analysis of renal protein expression levels of OATP1A1, OATP1A4 and MRP2. (F) Western blot analysis of nuclear extracts from liver and liver membranes for CAR determination. $\mathrm{n}=4$ in each group $\left({ }^{*} \mathrm{P}<0.05,{ }^{* * *} \mathrm{P}<0.01\right.$ and $\left.{ }^{* * * *} \mathrm{P}<0.001\right)$. CAR, constitutive androstane receptor; OATP, organic anion transporting polypeptide; UGT, UDP-glucuronosyltransferase; MRP, multidrug resistance-associated protein; TCP, 1,4-bis-[2-(3,5-dichlorpyridyloxy)]benzene; PB, pentobarbital; CO, corn oil; DS, dimethyl sulfoxide; RT-qPCR, reverse transcription-quantitative polymerase chain reaction.

two agonists, whereas the mRNA level of Mrp2 was induced 2-fold by TCP (Fig. 3D), with no clear difference in protein levels (Fig. 3E).

Effect of CAR ligands on the expression of CAR in a murine model of $A L D$. The present study also determined whether the expression of CAR was affected by CAR ligands in the ALD mice. The total liver mRNA and protein expression levels of CAR were not induced by TCPOBOP or PB treatment (Fig. 3B and F), as described previously (17). However, it was found that the protein expression of CAR in the nuclear extract was increased 1.6-fold following PB administration (Fig. 3F), as previously described (18). However, no significant alteration in the protein level of CAR was observed in the nuclear extract following TCP administration, compared with that of its vehicle.

In vitro subcellular localization of CAR. To further determine whether alcohol suppressed CAR translocation to the nucleus, the present study measured the expression of CAR using immunofluorescence analysis in normal mouse hepatocytes. As shown in Fig. 4, fluorescence was observed in the alcohol-treated cells, although few fluorescence dots were identified in the nuclei of the alcohol-treated cells. These 


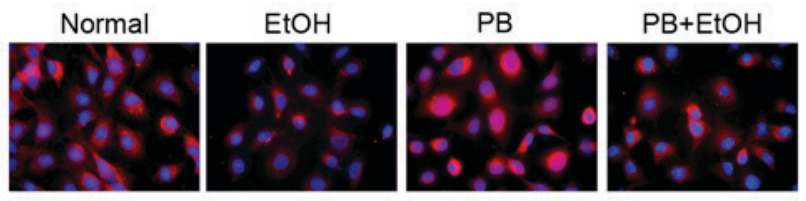

Figure 4. Immunofluorescence examination of CAR in hepatocytes Localization of CAR (red) via immunofluorescence microscopy (original magnification, $\mathrm{x} 400$ ) in normal, EtOH, $\mathrm{PB}$ and $\mathrm{PB}+\mathrm{EtOH}$ groups). CAR, constitutive androstane receptor; $\mathrm{PB}$, pentobarbital.

results indicated that alcohol impaired the PB-induced translocation of CAR from the cytoplasm to the nucleus.

\section{Discussion}

In the present study, plasma levels of bilirubin and the hepatic and extrahepatic bilirubin clearance pathways were examined in a murine model of ALD. It was found that chronic alcohol intake elevated plasma levels of bilirubin and induced the mRNA and protein levels of UGT1A1, which mediated the detoxification of bilirubin to less toxic substrates for biliary and urinary excretion, and induced alternative elimination pathways, including the MRP3 and MRP4 pathways. Despite the increased levels of UGT1A1, chronic alcohol consumption reduced the expression of hepatic MRP2, as reported by Zinchuk et al (22). Alcohol significantly reduced the transcriptional and post-transcriptional levels of OATP1A1 in the liver and kidney, which may have been a response to limit the cellular accumulation of bilirubin in the liver and the renal reuptake of bilirubin. Few reports have described the effects of ethanol on the reduction of OATP1A1. The altered levels of OATP1A1 and MRP2 may account for the pathological elevation of bilirubin in ALD, and the induction of UGT1A1 may be an adaptive response to the increased levels of bilirubin.

The present study also found that the protein levels of CAR in the nuclear extracts decreased with alcohol intake and increased with exposure to the CAR ligand, PB, which translocates CAR to the nucleus and causes the total liver protein to remain constant. This indicated that alcohol impaired CAR translocation. Furthermore, the distribution analysis of fluorescence in vitro confirmed that alcohol impaired the translocation of CAR and the PB-induced nuclear translocation of CAR. Hepatic OATPs, UGT1A1 and MRP2 represent CAR target genes, as previously described $(11,18,25)$. Therefore, alterations in transporters may be associated with the dysfunctional translocation of CAR and requires further investigation.

The nuclear translocation of CAR is a key step in inducer transactivation. Cytoplasmic CAR (NR1I3) forms heterodimers with the retinoid $\mathrm{X}$ receptor $\alpha$ (NR2B1) upon activation by its ligand to mediate binding with nuclear DNA response elements in the promoter regions, which is defined by different canonical hexamer sequences in the target genes (25). The phenobarbital-responsive enhancer module (PBREM) and the xenobiotic-responsive enhancer module have been identified as the CAR binding sites in response to CAR activation (13).

In order to further elucidate the effect of CAR on serum levels of bilirubin and the bilirubin clearance pathway in the murine model of ALD, the ALD mice were injected with the rodent-specific CAR agonist, TCPOBOP, and indirect CAR ligand, $\mathrm{PB}$, both of which promoted bilirubin clearance by decreasing plasma bilirubin levels (Table II). PB also induced the hepatic transcription of OATP1A1 and OATP1A4. The stimulatory effects of TCPOBOP on the expression levels of these two uptake transporters were moderate $(\mathrm{P}>0.05)$, whereas $\mathrm{PB}$ and TCPOBOP had no significant effect on OATP1B2. In the absence of mouse OATP1A and 1B transporters, the biliary excretion of conjugated bilirubin was reduced by $50 \%$. This finding indicated that, under physical conditions, at least half of the conjugated bilirubin was initially transported into the plasma, only to be taken up by hepatocytes downstream via OATP1A and 1B (26). Thus, the induction of OATP1A1 and OATP1A4 following the administration of CAR agonists explains the reduction in serum-conjugated bilirubin via the reuptake of bilirubin glucuronides (Table II). In addition, PB enhanced the transcriptional and post-transcriptional levels of UGT1A1, whereas TCPBOTOP stimulated the protein overexpression of UGT1A1 in the ALD murine model. UGT1A1, which is pivotal in the clearance of bilirubin, is the first glucuronidation enzyme targeted by CAR by binding to a distinct PBREM of UGT1A1 (27,28). TCPOBOP induced the expression of MRP2, consistent with a study by Petrick et al (17), however, MRP2 was less affected by PB. The induction of detoxification enzymes and the biliary excretion pump may also account for the accelerated serum bilirubin clearance following treatment with CAR agonists. The present study also detected renal transporters by administering these two ligands and found that OATP1A1 and OATP1A4 remained unaltered by the CAR ligands, and the mRNA expression of Mrp2 was induced by TCPOBOP, but not PB. This limited effect may be attributed to the lower renal expression of CAR, as previously described $(11,17)$. Similarly, previous studies have demonstrated that CAR agonists upregulate the expression levels of OATP2, UGT1A1 and MRP2 to inhibit cholestasis, with subsequent reduced serum levels of bilirubin, in a model of common bile duct ligation (11). Huang et al $(29,30)$ observed that TCPOBOP increased clearance under an acute dose of bilirubin, with induction of UGT1A1, MRP2, GSTA1 and GSTA2 in the bilirubin clearance pathway in the livers of wild-type, but not CAR-deficient, mice. Despite increased induction of its target gene, the expression of CAR was not induced by activators of CAR, as reported previously (17).

In the present study, in addition to protective effects as a bilirubin cleaner, CAR agonists also alleviated the degree of steatosis induced by alcohol, as shown using H\&E and oil red $\mathrm{O}$ staining. The CAR agonist, $\mathrm{PB}$, has been shown to improve lipid metabolism and enhance insulin sensitivity in diabetic rats (31). Furthermore, CAR agonists have been reported to inhibit the expression of lipogenic genes, including acetyl-CoA carboxylase (Acc-2) and stearoyl-CoA desaturase-1 (Scd-1), and rats deficient in Acc- 2 or Scd-1 are protected from hepatic steatosis induced by a high-fat diet (32). Similarly, CAR agonists alleviate steatosis of the liver by improving lipid metabolism, which requires further investigation. However, the CAR agonist, TCPOBOP, elevated the levels of ALT and AST, and enlarged hepatocytes by increasing the liver/body weight ratio as a side effect. Therefore, the selection of appropriate CAR activators is essential for bilirubin clearance in hepatocytes and for improved lipid metabolism. 
In conclusion, the results of the present study revealed that chronic alcohol consumption impaired the translocation of CAR, leading to defective bilirubin clearance accompanied by the reduction of hepatic and renal OATP1A1, the reduction of hepatic MRP2 and the induction of UGT1A1. The results also demonstrated that the activation of CAR by different ligands promotes bilirubin clearance by selectively inducing OATP1A1, OATP1A4, UGT1A1 and MRP2 in a murine model of ALD.

\section{Acknowledgements}

This study was supported by the Zhejiang Provincial Natural Science Foundation of China (grant nos. LY12H03003 and Y2110768).

\section{References}

1. Orman ES, Odena G and Bataller R: Alcoholic liver disease: Pathogenesis, management, and novel targets for therapy. J Gastroenterol Hepatol 28 (Suppl 1): S77-S84, 2013.

2. Chalmers DM, Rinsler MG, MacDermott S, Spicer CC and Levi AJ: Biochemical and haematological indicators of excessive alcohol consumption. Gut 22: 992-996, 1981.

3. Torkadi PP, Apte IC and Bhute AK: Biochemical evaluation of patients of alcoholic liver disease and non-alcoholic liver disease. Indian J Clin Biochem 29: 79-83, 2014.

4. Zollner G, Fickert P, Zenz R, Fuchsbichler A, Stumptner C, Kenner L, Ferenci P, Stauber RE, Krejs GJ, Denk H, et al: Hepatobiliary transporter expression in percutaneous liver biopsies of patients with cholestatic liver diseases. Hepatology 33: 633-646, 2001

5. Kardon T, Coffey MJ, Bánhegyi G, Conley AA, Burchell B, Mandl $\mathrm{J}$ and Braun L: Transcriptional induction of bilirubin UDP-glucuronosyltransrase by ethanol in rat liver. Alcohol 21: $251-257,2000$.

6. Flitman R and Worth MH Jr: Inhibition of hepatic alcohol dehydrogenase by bilirubin. J Biol Chem 241: 669-672, 1966.

7. Bodeman CE, Dzierlenga AL, Tally CM, Mulligan RM, Lake AD, Cherrington NJ and McKarns SC: Differential regulation of hepatic organic cation transporter 1 , organic anion-transporting polypeptide 1a4, bile-salt export pump and multidrug resistance-associated protein 2 transporter expression in lymphocyte-deficient mice associates with interleukin-6 production. J Pharmacol Exp Ther 347: 136-144, 2013.

8. Keppler D: The roles of MRP2, MRP3, OATP1B1, and OATP1B3 in conjugated hyperbilirubinemia. Drug Metab Dispos 42: $561-565,2014$

9. Erlinger S, Arias IM and Dhumeaux D: Inherited disorders of bilirubin transport and conjugation: New insights into molecular mechanisms and consequences. Gastroenterology 146: $1625-1638,2014$

10. Iusuf D, van de Steeg E and Schinkel AH: Functions of OATP1A and $1 \mathrm{~B}$ transporters in vivo: Insights from mouse models. Trends Pharmacol Sci 33: 100-108, 2012.

11. Wagner M, Halilbasic E, Marschall HU, Zollner G, Fickert P, Langner C, Zatloukal K, Denk H and Trauner M: CAR and PXR agonists stimulate hepatic bile acid and bilirubin detoxification and elimination pathways in mice. Hepatology 42: 420-430, 2005.

12. Yang H, Plösch T, Lisman T, Gouw AS, Porte RJ, Verkade HJ and Hulscher JB: Inflammation mediated down-regulation of hepatobiliary transporters contributes to intrahepatic cholestasis and liver damage in murine biliary atresia. Pediatr Res 66: 380-385, 2009.

13. Yang $\mathrm{H}$ and Wang $\mathrm{H}$ : Signaling control of the constitutive androstane receptor (CAR). Protein Cell 5: 113-123, 2014.

14. Tojima H, Kakizaki S, Yamazaki Y, Takizawa D, Horiguchi N, Sato K and Mori M: Ligand dependent hepatic gene expression profiles of nuclear receptors CAR and PXR. Toxicol Lett 212 : 288-297, 2012.
15. Luisier R, Lempiäinen $H$, Scherbichler N, Braeuning A, Geissler M, Dubost V, Müller A, Scheer N, Chibout SD, Hara H, et al: Phenobarbital Induces cell cycle transcriptional responses in mouse liver humanized for constitutive androstane and pregnane X receptors. Toxicol Sci 139: 501-511, 2014.

16. Assenat E, Gerbal-Chaloin S, Larrey D, Saric J, Fabre JM, Maurel P, Vilarem MJ and Pascussi JM: Interleukin 1beta inhibits CAR-induced expression of hepatic genes involved in drug and bilirubin clearance. Hepatology 40: 951-960, 2004.

17. Petrick JS and Klaassen CD: Importance of hepatic induction of constitutive androstane receptor and other transcription factors that regulate xenobiotic metabolism and transport. Drug Metab Dispos 35: 1806-1815, 2007.

18. Saini SP, Mu Y, Gong H, Toma D, Uppal H, Ren S, Li S, Poloyac SM and Xie W: Dual role of orphan nuclear receptor pregnane $\mathrm{X}$ receptor in bilirubin detoxification in mice. Hepatology 41: 497-505, 2005.

19. Chen X, Meng Z, Wang X, Zeng S and Huang W: The nuclear receptor CAR modulates alcohol-induced liver injury. Lab Invest 91: 1136-1145, 2011.

20. Wu W, Zhu B, Peng X, Zhou M, Jia D and Gu J: Activation of farnesoid $X$ receptor attenuates hepatic injury in a murine model of alcoholic liver disease. Biochem Biophys Res Commun 443: 68-73, 2014

21. Fisher CD, Lickteig AJ, Augustine LM, Oude Elferink RP, Besselsen DG, Erickson RP and Cherrington NJ: Experimental non-alcoholic fatty liver disease results in decreased hepatic uptake transporter expression and function in rats. Eur J Pharmacol 613: 119-127, 2009.

22. Zinchuk V, Zinchuk O, Akimaru K, Moriya F and Okada T: Ethanol consumption alters expression and colocalization of bile salt export pump and multidrug resistance protein 2 in the rat. Histochem Cell Biol 127: 503-512, 2007.

23. Lee J, Azzaroli F, Wang L, Soroka CJ, Gigliozzi A, Setchell KD, Kramer W and Boyer JL: Adaptive regulation of bile salt transporters in kidney and liver in obstructive cholestasis in the rat. Gastroenterology 121: 1473-1484, 2001.

24. Masereeuw R and Russel FG: Regulatory pathways for ATP-binding cassette transport proteins in kidney proximal tubules. AAPS J 14: 883-894, 2012.

25. Geier A, Wagner M, Dietrich CG and Trauner M: Principles of hepatic organic anion transporter regulation during cholestasis, inflammation and liver regeneration. Biochim Biophys Acta 1773: 283-308, 2007.

26. van de Steeg E, Wagenaar E, van der Kruijssen CM, Burggraaff JE, de Waart DR, Elferink RP, Kenworthy KE and Schinkel AH: Organic anion transporting polypeptide $1 \mathrm{a} / 1 \mathrm{~b}-\mathrm{knockout}$ mice provide insights into hepatic handling of bilirubin, bile acids, and drugs. J Clin Invest 120: 2942-2952, 2010.

27. Sugatani J, Kojima H, Ueda A, Kakizaki S, Yoshinari K, Gong QH, Owens IS, Negishi M and Sueyoshi T: The phenobarbital response enhancer module in the human bilirubin UDP-glucuronosyltransferase UGT1A1 gene and regulation by the nuclear receptor CAR. Hepatology 33: 1232-1238, 2001

28. Tolson $\mathrm{AH}$ and Wang $\mathrm{H}$ : Regulation of drug-metabolizing enzymes by xenobiotic receptors: PXR and CAR. Adv Drug Deliv Rev 62: 1238-1249, 2010.

29. Huang W, Zhang J, Chua SS, Qatanani M, Han Y, Granata R and Moore DD: Induction of bilirubin clearance by the constitutive androstane receptor. Proc Natl Acad Sci USA 100: 4156-4161, 2003.

30. Huang W, Zhang J, Chua SS, Qatanani M, Han Y, Granata R and Moore DD: Induction of bilirubin clearance by the constitutive androstane receptor (CAR). Proc Natl Acad Sci USA 100: 4156-4161, 2003.

31. Venkatesan N, Davidson MB, Simsolo RB and Kern PA: Phenobarbital treatment enhances insulin-mediated glucose metabolism and improves lipid metabolism in the diabetic rat. Metabolism 43: 348-356, 1994.

32. Gao J, He J, Zhai Y, Wada T and Xie W: The constitutive androstane receptor is an anti-obesity nuclear receptor that improves insulin sensitivity. J Biol Chem 284: 25984-25992, 2009. 